\title{
High Precision Low Temperature Coefficient Current Reference with Resistor Compensation
}

\author{
Xiaoning Xin, Bingyin Luo \\ School of Information Science and Engineering \\ Shenyang university of technology, shenyang,110870, China \\ E-mail:xinxn2007@yahoo.com.cn
}

\begin{abstract}
In order to improve temperature characteristics and precision of current reference, a novel resistor compensated current reference based on bandgap voltage reference with trimming network is proposed in this paper. By introducing the combination of 2 nd order resistor and transmission gate whose temperature characteristics compensate that of bandgap voltage reference, lower temperature coefficient has been achieved. This circuit is implemented using HHNEC 0.35um BCD process with $3.3 \mathrm{~V}$ power supply. Simulation results for the proposed current reference show the worst temperature coefficient of $15.25 \mathrm{ppm} /{ }^{\circ} \mathrm{C}$ and the best temperature coefficient of $5 \mathrm{ppm} /{ }^{\circ} \mathrm{C}$ over a temperature range from $-45^{\circ} \mathrm{C}$ to $85^{\circ} \mathrm{C}$. With trimming network, an output current variation of $1.4 \%$ is achieved among process corners. On the premise of not introducing high-order curvature compensated to bandgap which increase circuit complexity, this circuit put forward a new thought for the design of current reference.
\end{abstract}

Keywords-Current reference, Resistor compensation, Trimming network, Temperature coefficient

\section{INTRODUCTION}

Current reference is a basic building block in analog circuit. The implementation of analog-mixed circuit such as current-steering data converters, loop-powered data converters[1], etc. needs stable bias current which is derived from current reference. With layout techniques to improve matching, reference current can be accurately mirrored and multipled by current mirrors, then bias other components in analog circuit. So it is of great significance to design a high precision low temperature coefficient current reference.

It has been admitted that the current reference does not exist alone but has to consist of a bandgap voltage reference (BVR) and one or two resistors. With curvature correction and resistor trimming technology, the temperature coefficient (TC) of BVR could be kept under $2 \mathrm{ppm} /{ }^{\circ} \mathrm{C}$ [2][3][4]. But the corresponding low TC current references have been rarely covered. The major difficulty is the lack of resistor that is independent to temperature[5], which means the bandgap voltage reference is not virtually irrelevant to temperature when designing low TC current reference. By compromising parts of BVG temperature coefficient to cooperate with resistor, we obtain better current reference. Based on study of traditional current reference, we propose a novel resistor-compensated current reference to achieve better temperature coefficient. By adding resistor trimming network to the circuit, high precision is expected to be fulfilled.

\section{TRADITIONAL CURRENT REFERENCE}

In order to get current reference from BVR, a resistor is needed[6]. The best way converting voltage to current is using amplyfier. The schematic of traditional current reference has been shown in Figure.1. The circuit uses an amplifier in a negative feedback loop to drive the voltage across resistor $\mathrm{R}$ euqal to bandgap reference voltage $V_{R E F}$. The current $I_{\text {OUT }}$ flowing through current regulator MOS $P$ is forced to be exactly the same with current flowing through resistor $R$. The major error comes from input offset voltage of amplyfier $A M P$.

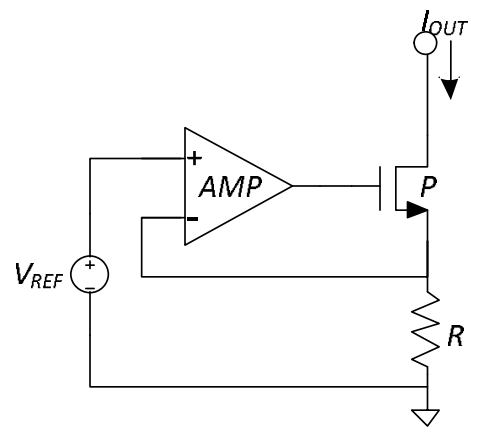

Figure 1. Traditional current reference

When voltage reference $V_{R E F}$ and the resistor $R$ are all ideal, output current can be expressed as $I_{O U T}=V_{R E F} / R$. But the integrated resistors which are related to temperaure and process make current reference highly sensitive to temperature change and process variation. This problem can be solved perfectly by using off-chip hybrid resistor[7], but hybrid resistor is costly and cannot be implemented in off-chip-resistor-prohibited system. Hosung Chun proposed a method that can reduce the effect of resistor value variations by combining different kinds of integrated resistor together in series[8]. Each kind of integrated resistor has its own process parameter independent to others. When the process changes, different kinds of resistor can compensate each other to achieve stable resistence.

The methods mentioned above make current reference more precise, but they all ingored the resistor's own temperature characteristics which affect temperature characteristics of current reference. One way to solve this problem is adding $I_{P T A T}$ and $I_{\text {CTAT }}$ current to compensate 
high-order term in current reference[9]. Ming-Dou Ker[10] reports utlizling the difference of temperature-dependent currents generated from the parasitic $n-p-n$ and $p-n-p$ bipolar junctions to obtain temperaure-independent current reference. All these methods ignore the resistor temperature characteristics and require complex circuits implementation.

Temperature characteristics of $1^{\text {st }}$ order compensated BVR are slightly parabolic shape, where output voltage has a nearly zero temperature coefficient at peak. As the current reference is the output voltage of BVR divided by the resistor, therefore we conside to design a resistor whose temperature characteristics compensate the curve of BVR to achieve better TC current reference. The compensation theory is shown in Figure.2.

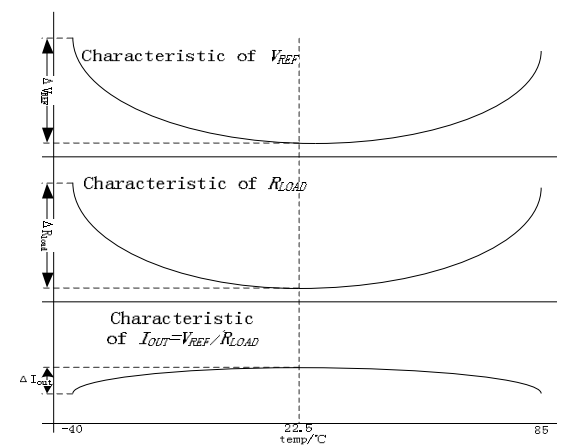

Figure 2. The theory of resistance compensation

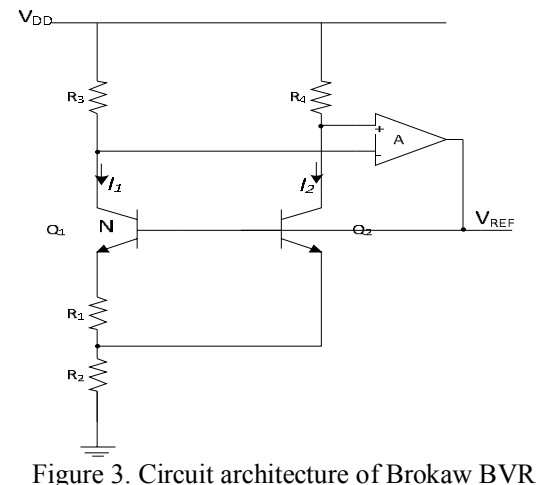

III. CHARACTERASTICS OF BANDGAP VOLTAGE

\section{REFERENCE}

A voltage reference with zero temperature coefficient can be achieved by adding the negative TC of $V_{B E}$ to positive TC $V_{P T A T}$ weighted by $K$, since the voltages referenced to $V_{B E}$ and $V_{P T A T}$ have opposite temp coefficient.

$$
V_{\text {REF }}=V_{B E}+K \times V_{\text {PTAT }}
$$

Based on theory above, many BVR circuits have been proposed. Among them, the Brokaw bandgap[11] is widely used in high precision data convertors for its simplicity, ability to operate at low supply voltages and easing of scaling the output voltage above the silcon bandgap voltage. The circuit archtecture of Brokaw BVR is shown in Figure.3.
In this circuit, amplifier $A$ in deep negative feedback loop enforces the voltage across resistor $R_{3}, R_{4}$ to be the same voltage. If $R_{3}$ is equal to $R_{4}$, the bias currents $I_{1}$ and $I_{2}$ flowing through collector of n-p-n bipolar $Q_{1}$ and $Q_{2}$ are identical. Assuming the current gain of $n-p-n$ bipolar is large enough that base current of n-p-n bipolar can be ignored, we obtain:

$$
\mathrm{V}_{\text {REF }}=V_{B E 2}+\left(I_{1}+I_{2}\right) R_{2}
$$

Because the emitter area of $Q_{1}$ is $\mathrm{N}$ times larger than that of $Q_{2}$, with the same biased current the value of $V_{B E Q 1}$ is smaller than $V_{B E Q 2}$. The difference bewteen these two voltage is the proportional to absolute temperature voltage $\left(V_{P T A T}\right)$. Dividing $V_{P T A T}$ by resistor $R_{l}$ we get the expression $I_{P T A T}$ :

$$
\begin{aligned}
& I_{P T A T}=\frac{V_{B E 2}-V_{B E 1}}{R_{1}}=\frac{V_{T} \ln \frac{I_{C 2}}{I_{S 2}}-V_{T} \ln \frac{I_{C 1}}{I_{S 1}}}{R_{1}} \\
& =\frac{V_{T}}{R_{1}} \ln \frac{I_{C 2} I_{S 1}}{I_{C 1} I_{S 2}}=\frac{k T}{R_{1} q} \ln N=\frac{\beta}{R_{1}} \cdot T
\end{aligned}
$$

Where $V_{B E 2}$ is the forward voltage drop across the base emitter junction of bipolar $Q_{2}$. The relationship between $V_{B E}$ and temperature is a nonlinear property, Tsividis studied Temp- $V_{B E}$ characteristic of bipolar and gave the euqation as[12]

$$
\begin{aligned}
V_{B E} & =V_{\mathrm{g} 0}-\left(\frac{T}{T_{0}}\right) \cdot\left(V_{\mathrm{g} 0}-V_{B E}\left(T_{0}\right)\right)-(\eta-\alpha) \frac{k T}{q} \ln \left(\frac{T}{T_{r}}\right) \\
= & V_{\mathrm{g} 0}-\left[\frac{V_{\mathrm{g} 0}-V_{B E}\left(T_{0}\right)}{T_{0}}-\frac{k(\eta-\alpha)}{q} \cdot \ln T_{0}\right] \cdot T-\left[\frac{k(\eta-\alpha)}{q}\right] \cdot T \ln T \\
= & \alpha_{0}-\alpha_{1} T-\alpha_{2} \cdot T \cdot \ln T
\end{aligned}
$$

Where $V_{g 0}$ is the bandgap voltage of silicon at $0^{\circ} \mathrm{K}, \quad \eta$ is a constant depending on process, $a$ is the order of tempature dependence of collect bias current, when the current flows through bipolar is small and proportational to absolute temperaure $a=1$, otherwise $a=1$. In (4), the term of $T \ln T$ can be expanded by Taylor series, we obtain: $T \ln T$ $\approx T^{2} / 2-1 / 2$, rewrite (4):

$$
V_{\mathrm{BE}}=\left(\alpha_{0}+\frac{\alpha_{2}}{2}\right)-\alpha_{1} T-\frac{\alpha_{2}}{2} T^{2}
$$

Substitude (3) (5) into (2), we can get the expression of Brokaw BVR:

$$
\begin{aligned}
& V_{R A F}=V_{B E}+V_{P T A T}=\left(\alpha_{0}+\frac{\alpha_{2}}{2}\right)-\alpha_{1} T-\frac{\alpha_{2}}{2} T^{2}+2 \beta \frac{R_{2}}{R} \cdot T \\
& =\left(\alpha_{0}+\frac{\alpha_{2}}{2}\right)-\left(\alpha_{1}-2 \beta \frac{R}{R}\right) T-\frac{\alpha_{2}}{2} T^{2}
\end{aligned}
$$

Expression (6) indicates that the temperature characteristics of $1^{\text {st }}$ order compsenstaed Brokaw BVR has parabolic shape. The location of the peak voltage of $V_{R E F}$ is a function of the ratio of $R_{1}$ to $R_{2}$. This relationship can be derived by first recognizing that $V_{R E F}$ peaks at $T_{0}$, where 
$d V_{R E F} / d T=0$. Solving this equation for $T$, substituting $T$ with $T_{0}$, and solving for $R_{1} / R_{2}$, yields the resistor ratio that will place the peak of $V_{R E F}$ at any specified $T_{0}$. [13] The equation is:

$\frac{R_{2}}{R_{1}}=\frac{\alpha_{1}-\alpha_{2} T_{0}}{2 \beta}$

$T_{0}$ where $d V_{R E F} / d T=0$ is called the "Magic Temperature". From the nature of parabola, only when the magic temperature point locates in the middle of full temperature range, can we get the minimum difference of $V_{R E F}$ to achieve the best temperautre coefficient in the condition of $1^{\text {st }}$ order compensate.

\section{DISSCUSSION ON RESISTOR COMPENSATION}

In CMOS process, the resistance varies with the temperature and the voltage applied on it. SPICE models use parameter TCR and VCR to represent temperature coefficient and voltage coefficient respectively. In SPICE models, resistance is defined as[14]

$$
R_{\text {eff }}=R_{S H 0} \frac{L}{W_{\text {eff }} p f} \times v c_{\text {coeff }} \times\left[1+T C R 1 \cdot T+T C R 2 \cdot T^{2}\right]
$$

Where $p f$ is the numbers of multiple fingers, $L$ is the length of resistor, $W_{\text {eff }}$ is the effective width deducting beak effect, $R_{S H O}$ is sheet resistance measured at $0^{\circ} \mathrm{C}$. The $R_{S H}$ is proportinal to resistivity $\rho$ which is a temperature dependent variable[15], SPICE uses a quadratic to model the temperature dependence of a resistor, TCR 1 is the $1^{\text {st }}$ order temperature coefficient whereas TCR 2 is the $2^{\text {nd }}$ order temperature coefficient. If we choose the resistor with $2^{\text {nd }}$ order temperature coefficient in process whose temerature characteristics match Brokaw BVR temperature characterisitcs, it is possbile to achieve lower temperature coefficient current reference. In practice, due to process limitation, it is difficult to find a resistor whose temperature characteristics perfectly match that of BVR. We should compensate this $2^{\text {nd }}$ order resister to make it matches with the BVR better.

The simplest way of compensating $2^{\text {nd }}$ order resistor is to connect $1^{\text {st }}$ order resistor connecting in series. The resistance of $1^{\text {st }}$ order resistor compensated hybird resistor can be expressed as:

$$
\begin{aligned}
& R=R_{1 s t}+R_{2 n d}=R_{1} \cdot\left(1+T C R 1_{1} \times T\right)+R_{2} \cdot\left(1+T C R 1_{2} \times T+T C R 2_{2} \times T^{2}\right) \\
& =R_{2} T C R 2_{2}\left(T+\frac{R_{1} T C R 1_{1}+R_{2} T C R 1_{2}}{2 R_{2} T C R 2_{2}}\right)^{2}+C
\end{aligned}
$$

The temperature characterisitcs of $1^{\text {st }}$ order resistor compensation is parabolic. The curve needed to compensate Brokaw BVR can be obtained by adjusting the ratio of $R_{l}$ to $R_{2}$. But this method consumes large layout area and cannot be compatible with trimming technology which guarantees the precision of current reference. Transmission gate(TG) consisting of NMOS and PMOS whose resistance is the MOS on-resistance is a better choice. In switching state, the resistance of TG is defined as the ratio of drain-source voltage $\left(V_{D S}\right)$ to drain current $I_{D}$ :

$$
R_{O N} \equiv \frac{V_{D S}}{I_{D}}=\frac{L \cdot t_{o x}}{\mu_{n} \cdot \varepsilon_{o x} \cdot W \cdot\left(V_{G S}-V_{T}\right)}
$$

Where $V_{T}$ is the threshold of MOS which has a linear relationship with temperature, $\mu_{n}$ is the mobility of electron whose temperature characteristic is proportional $T^{1.5}$. Simulation results show that the on-resistance of TG approximately has a linear relationship with temperature[16]. Therefore ratio of width to length of MOS in TG can be adjusted so that the temperature characteristics of TG compensated hybrid resistor match that of the Brokaw BVR, which is the similar to $1^{\text {st }}$ order resistor compensated method. Compared with $1^{\text {st }}$ order resistor compensation, layout area can be reduced by using TG. More importantly $\mathrm{TG}$ can be used in digital trimming circuit to improve the precision of current reference.

\section{PROPOSED CIRCUIT ARCHITECTURE}

The schematic of proposed CMOS current reference is shown in Figure.4. The core circuit consists of two parts: $2.5 \mathrm{~V}$ bandgap voltage reference and $32 \mathrm{uA}$ current reference. By means of an operational amplifier and transistors, a stable voltage reference $V_{R E F}$ generated by Brokaw BVR is applied across a compensated resistor which consists of a $2^{\text {nd }}$ order resistor and a TG connecting in series, resulting in a current. Bandgap reference biasing circuit and start-up circuit are also added to make current reference a complete system.

The core of $2.5 \mathrm{~V}$ Brokaw BVR consists of $Q_{8}, Q_{9}, R_{6}$, $R_{7}$. The error amplifier which consists of $P_{5}, P_{6}, Q_{11}, Q_{12}$ and $R_{8}$ enforces collectors of $Q_{8}, Q_{9}$ to have equal potential. The $2.5 \mathrm{~V}$ Brokaw BVR is supplied by current source composed of $P_{7}$ and $Q_{10}$. Compared with voltage supply, current supply can improve the current reference's PSRR by obtaining feedback current from BVR rather than a voltage. $R_{9}$ and $R_{10}$ are used to boost the output voltage of BVR. Ignoring the input offset of error amplifier we know $V_{R E F}=V_{l}\left(1+R_{10} / R_{9}\right)$, where $V_{1}=1.25$ is the Brokaw BVR output voltage. Making $R_{10}=R_{9}$, we obtain $2.5 \mathrm{~V}$ voltage reference $V_{R E \mathrm{~F}}$.

In $32 \mathrm{uA}$ current reference circuit, $P_{10}, P_{11}, Q_{14}, Q_{15}, Q_{16}$, $R_{15}$ and $P_{12}, N_{3}$ compose of a two-stage amplifier used to increase the output resistance of current reference. Bias circuit consists of $P_{8}, P_{9}, Q_{13}, R_{14}$ and $N_{2}$, and the bias voltage is supplied by $1.25 \mathrm{~V}$ bandgap bias which consists of $P_{3}, P_{4}, Q_{5}, Q_{6}$ and $R_{2}, R_{3}$. The two-stage amplifier is in deep feedback state, so voltages apply on differential $\operatorname{pairs}\left(V_{+}\right.$and $\left.V_{-}\right)$are forced to be equal: $V_{+}=V_{-}=V_{R E F}{ }^{\bullet}$ $R_{14} /\left(R_{11}+R_{14}\right)$. If $R_{11}=R_{17}$, then we have:

$$
I_{\text {out }}=\frac{V_{R E F}-V_{+}}{R_{17}}=\frac{V_{R E F}}{R_{11}+R_{14}}
$$

The Brokaw BVR has a zero-state when there is no current in the circuit at start-up. Therefore start-up circuits are introduced to avoid zero-state at start-up. $P_{1}, Q_{1}, Q_{2}, Q_{3}$ and $R_{l}$ compose the $1.25 \mathrm{~V}$ bandgap bias start-up circuit. $P_{l}$ 
is a long channel PMOS used as MOS resistor. When the power is on, start current flows through $P_{1}, Q_{2}$ to the core of $1.25 \mathrm{~V}$ Brokaw BVR. Once the BVR is on, the $\triangle V_{B E 2}$ becomes less than $0.7 \mathrm{~V}$ turning off the $Q_{2}$.The start-up process is over. $2.5 \mathrm{~V}$ BVR start-up circuit consists of $P_{2}, Q_{4}$, $Q_{7} .1 .25 \mathrm{~V}$ bandgap bias provides a start-up current flowing through $P_{2}$ and $Q_{4}$ to the core of $2.5 \mathrm{~V}$ BVR. Similarly, once $2.5 \mathrm{~V} \mathrm{BVR}$ is on, the $\Delta V_{B E 7}$ becomes lower than $0.7 \mathrm{~V}$ turning off the start-up circuit of $2.5 \mathrm{~V}$ BVR.

\section{SIMULATION RESULTS AND ANALYSIS}

Based on HHNEC035um BCD process, the current reference circuit is simulated with HSPICE. Figure. 5 shows the current reference output $I_{O U T}$ versus temperature at TT corner. At TT corner the maxim value of current is $32.01 \mathrm{uA}$ at $22.5^{\circ} \mathrm{C}$, and it has the minimum value $31.97 \mathrm{uA}$ at $-40^{\circ} \mathrm{C}$ and $85^{\circ} \mathrm{C}$. The curve is totally a parable shape as expected with the TC of $11.25 \mathrm{ppm} /{ }^{\circ} \mathrm{C}$. Figure.6 shows the $2.5 \mathrm{~V}$ BVR output $V_{R E F}$ versus temperature, the zero-drift point is around $22.5^{\circ} \mathrm{C}$, the TC of BVR is $7.057 \mathrm{ppm} /{ }^{\circ} \mathrm{C}$. We get the resistance versus temperature from $R_{L O A D}=V_{R E F} / I_{O U T}$, as shown in Figure.7. From the curve, we found that the transmission gates compensate the $2^{\text {nd }}$ order resistor perfectly. The total power dissipation is $0.42 \mathrm{~mW}$ at $3.3 \mathrm{~V}$ voltage supply.

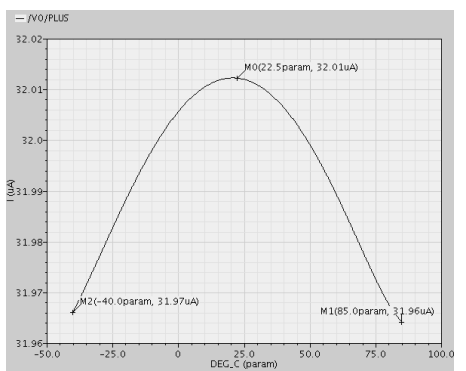

Figure 5. current vs. temperature

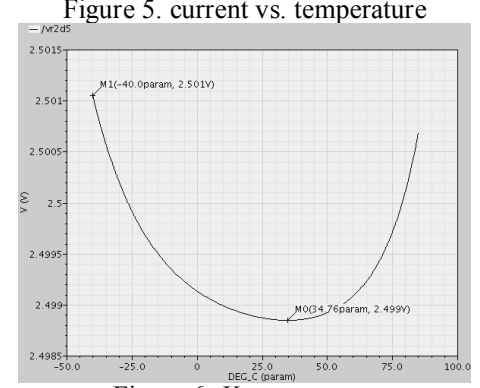

Figure $6 . V_{R E F}$ vs. temperature

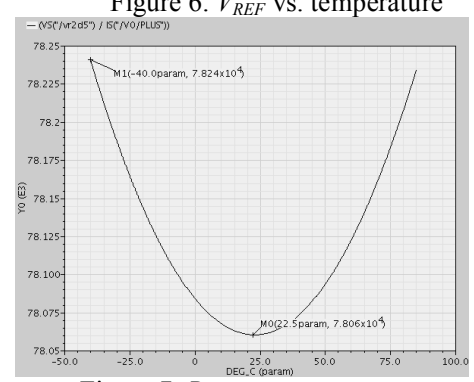

Figure 7. $R_{L O A D}$ Vs.temperature
Because the resistance varies $\pm 20 \%$ with process changing, digital trimming technology was introduced to guarantee the precision requirement. With transmission gates, the $R_{14}$ in Figure. 4 was divided into serval binary weighted segements. The transmission gates are controlled by serial shift register composed of d-flip-flops connecting in series. The schematic diagram is shown in Figure.8. The ratio of $W$ to $L$ of each transmission gate must be set carefully, so that the temperature characteristic of each segements match the temperature characteristic of BVR.

During the simulation, we set the processes of resistor independent to other device. After trimming, the current reference outputs $\left(I_{O U T}\right)$ and bandgap output voltages $\left(V_{R E F}\right)$ at 9 corner are presented in Tab.1:

The worst temperature coefficient (TC) of current reference which is about $15.25 \mathrm{ppm} /{ }^{\circ} \mathrm{C}$ appears in fast-fast corner of other device and typical resistor process corner. In the case of slow-slow of other device where the temperature characteristic of resistor matches $V_{R E F}$ better, although the TC of $V_{R E F}$ is worse, the TC of $I_{O U T}$ is better and can achieve as low as $5 \mathrm{ppm} /{ }^{\circ} \mathrm{C}$.

\section{CONCLUSION}

The resistor compensated accurate current reference with digital trimming technology is proposed. Under the condition of not introducing high order compensation to bandgap, we use the combination of transmission gate and $2^{\text {nd }}$ order resistor compensating the tepmerature characteristic of Brokaw bandgap reference. To calibre the process violation, the serial shift registers change the value of resistor network which controls the temperature coefficient and magnitude of the reference current perfectly. Simulation result showed the worst temperature coefficient of $15.25 \mathrm{ppm} /{ }^{\circ} \mathrm{C}$ for the reference current across a temperature range from $-40^{\circ} \mathrm{C}$ to $85^{\circ} \mathrm{C}$ and the maximum output reference variation of $1.5 \%$ among nine process corners.

\section{REFERENCES}

[1] WANG Xian-zhong, LIU Wei, HE Shu-kai. Application of AD421 in Smart Transmitter. [J]. INSTRUMENT TECHNIQUE AND SENSOR, 2006, (3):49 51

[2] ZHOU Ze-kun, MA Ying-qian, MING Xin, et al. A 2.2-V 2.9-ppm $/{ }^{\circ} \mathrm{C}$ BiCMOS bandgap voltage reference with full temperature-range curvature-compensation. [J]. Journal of Semiconductors, July 2010:Vol.31, No.7

[3] LU Yang, ZHANG Bo. A $1.8-\mathrm{V} \quad 0.7 \mathrm{ppm} /{ }^{\circ} \mathrm{C}$ high order temperature-compensated CMOS current reference. [J]. Analog Integr Circ Sig Process (2007) 51:175-179

[4] LU Shen, NING Ning, QI Yu.New Curvature Compensated CMOS Bandgap Voltage Reference. [J]. JOURNAL OF ELECTRONIC SCIENCE AND TECHNOLOGY OF CHINA, Dec 2007:VOL5 No.4

[5] Phillip E.Allen, Douglas R.Holberg. CMOS Analog Circuit Design (Second Edition). Beijing: Electronic industry press, 2005.

[6] Willy Willy M.C. Sansen. Analog Design Essentials [M]. Beijing: Tsinghua university press, 2008. 
[7] ZHANG Xin-wang, MENG Hai-tao, DU Zhan-kun. Sub- $1 \times 10-6 /{ }^{\circ} \mathrm{C}$ Low Voltage CMOS Bandgap Current Reference [J]. Design and Development of IC, 2010, 35(5):485-488

[8] Hosung Chun, Torsten Lehmann. CMOS Current Reference Generator Using Integrated Resistors. ICEIE 2010 [C]. Kyoto, Japan, August 1-3, 2010

[9] Byung-Do Yang, Young-Kyu Shin, Jee-Sue Lee, et al. An Accurate Current Reference using Temperature and Process Compensation Current Mirror. IEEE Asian Solid-State Circuits Conference[C] November 16-18, 2009 / Taipei, Taiwan

[10] Ming-Dou Ker, Jung-Sheng Chen. New Curvature Compensation Technique for CMOS Bandgap Reference With Sub-1-V Operation[J]. IEEE TRANSACTIONS ON CIRCUITS AND SYSTEMS, August 2006: VOL. 53, NO. 8 Bandgap Reference With Sub-1-V Operation
[11] Brokaw, A.P. A simple three-terminal IC bandgap reference. [J]. IEEE Journal of Solid-State Circuits, Dec, 1974: VOL. 9 Issue: 6:388-393.

[12] Tsividis, Y. P. Accurate analysis of temperature effects in IC-VBE characteristics with application to bandgap reference sources. [J]. IEEE Journal of Solid-State Circuits, 15, 1076-1084.

[13] Stephen D. Edwards. BANDGAP REFERENCE CALCULATOR USER'S GUIDE. Jan 26, 2011.

[14] R. Jacob Baker. CMOS Circuit Design, Layout, and Simulation (Second Edition). [M]. Beijing: POSTS \& TELECOM PRESS, 2008.

[15] LIU En-ke, ZHU Bing-sheng, LUO Jin-sheng. Semiconductor physics $[\mathrm{M}]$. (Fourth Edition) Beijing: National defense industrial press, 2009.

[16] CHEN Xin-bi, ZHANG Qing-zhong. Principle and design of transistor (Second Edition) $[\mathrm{M}]$.Beijing: Electronic industry press, 2008

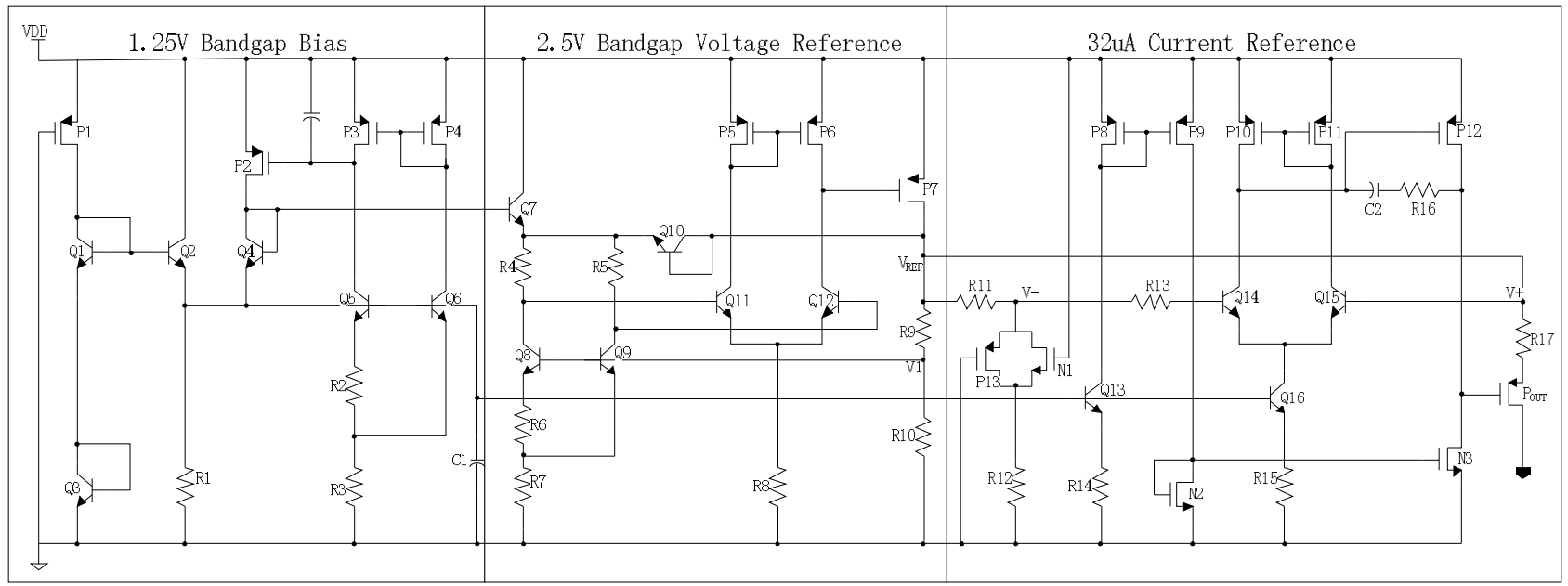

Figure 4. Proposed circuit archicture

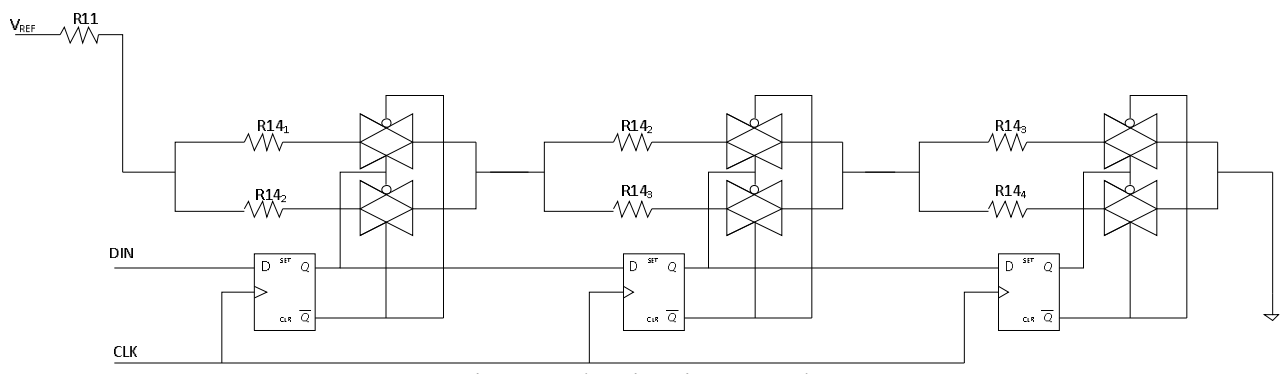

Figure 8 . The trimming network

TABLE I. OUTPUT OF CERRENT REFERENCE AND BVR AT 9 CORNERS

\begin{tabular}{cccccccc}
\hline $\begin{array}{c}\text { other device } \\
\text { corner }\end{array}$ & $\begin{array}{c}\text { resistor } \\
\text { corner }\end{array}$ & $\begin{array}{c}\boldsymbol{V}_{\text {REF }} \\
\text { min }\end{array}$ & $\begin{array}{c}\boldsymbol{V}_{\text {REF }} \\
\mathbf{m a x}\end{array}$ & $\begin{array}{c}\boldsymbol{I}_{\text {OUT }} \\
\text { min }\end{array}$ & $\begin{array}{c}\boldsymbol{I}_{\text {OUT }} \\
\text { max }\end{array}$ & $\begin{array}{c}\boldsymbol{I}_{\text {OUT }} \\
\text { precision }\end{array}$ & $\begin{array}{c}\text { IoUT } \\
\text { TC }\end{array}$ \\
\hline $\mathrm{TT}$ & $\mathrm{TT}$ & 2.4988 & 2.5011 & 31.965 & 32.013 & $1.0 \%$ & 11.25 \\
$\mathrm{TT}$ & $\mathrm{MAX}$ & 2.4984 & 2.5006 & 31.969 & 32.029 & $0.9 \%$ & 15.00 \\
$\mathrm{TT}$ & $\mathrm{MIN}$ & 2.4982 & 2.5016 & 31.979 & 32.035 & $1.1 \% 0$ & 14.00 \\
$\mathrm{FF}$ & $\mathrm{TT}$ & 2.4968 & 2.5000 & 31.970 & 32.031 & $1.0 \% 0$ & 15.25 \\
$\mathrm{FF}$ & $\mathrm{MAX}$ & 2.4988 & 2.5012 & 31.963 & 32.020 & $1.1 \% 0$ & 14.25 \\
$\mathrm{FF}$ & $\mathrm{MIN}$ & 2.4984 & 2.5028 & 31.987 & 32.047 & $1.4 \%$ & 15.00 \\
$\mathrm{SS}$ & $\mathrm{TT}$ & 2.4984 & 2.5033 & 31.987 & 32.007 & $0.4 \%$ & 5.000 \\
$\mathrm{SS}$ & $\mathrm{MAX}$ & 2.5000 & 2.5047 & 31.998 & 32.019 & $0.6 \%$ & 5.250 \\
$\mathrm{SS}$ & $\mathrm{MIN}$ & 2.4978 & 2.5034 & 31.990 & 32.019 & $0.6 \% 0$ & 7.250 \\
\hline
\end{tabular}

